\title{
Teaching hand-washing with pictorial cues ${ }^{1}$
}

\author{
Timo Saloviita ${ }^{2}$
}

\begin{abstract}
Applied behavior analysis has been shown to be an effective means to teach daily living skills to individuals with intellectual disability. In the present study pictorial cues based on task analysis, system of least prompts, and social reinforcement were used to teach a man with mild intellectual disability to wash his hands correctly. An ABAB reversal design was used with follow-up after two weeks. The results show a rapid increase in hand-washing skills.
\end{abstract}

Keywords: grooming behavior; intellectual disabilities; ABAB-design; task analysis; system of least prompts

\section{Introduction}

The use of teaching methods which give an opportunity for the learner to participate in his own instruction is an important tool for empowering students with disabilities (Browder \& Snell, 1993). Self-management methods are especially appropriate in the learning of daily living and community skills because we all use them in this context. For example, people typically use written aids to assist them in daily routines like shopping. Use of pictures provides a good form of assistance for people who have learning difficulties. Using pictorial cues, students with intellectual disabilities have been taught to prepare foods (Martin, Rusch, James, Decker, \& Trytol, 1982), to learn child-care skills Feldman, 2004; Feldman, Ducharme, \& Case, L. (1999), to function more independently in a job setting (Connis, 1979) or in leisure activities (Bambara \& Ager, 1992), to initiate and maintain grooming behaviours (Thinesen \& Bryan, 1981), to develop pedestrian navigations skills (Kelley, 2012) and withdraw money and purchase items using a debit card (Alberto, Cihak, \& Gama, 2005). Pictorial cues have been found to be more effective than verbal cues in promoting functional activities among children with disabilities (Caffó, Albano, Damato, \& Stella, 2013; West, 2008). They also facilitate acquisition of generalized skills (Phillips \& Vollmer, 2012). Besides intellectual disabilities, picture cues have been successfully used for persons with Alzheimer disease (Lancioni et al. 2012). The aim of the present study was to investigate the use of pictorial cues in the acquisition of hand-washing skills of a man with mild intellectual disabilities.

\section{Method}

\section{Participant and setting}

The participant of this study was a 27 -year-old man with mild intellectual disability and epilepsy. He could read, write, and count. He lived with his parents and daily went to sheltered

\footnotetext{
${ }^{1}$ Irene H. Karanka and Marianne Tuulkari assisted in the data collection of this study

${ }^{2}$ Ph.D., University of Jyvaskyla, Faculty of Education, Department of Teacher Education, timo.saloviita@jyu.fi
} 
workshop. Before the training began, he washed his hands by simply keeping them under running water, and then inefficiently wiping them dry. The study was done in the bathroom of the sheltered workshop.

\section{Target behaviour and task analysis}

Hand-washing as a target behaviour was broken down into 9 steps. The steps were as follows: goes to wash-basin, turns the water tap on, wets the hands, takes soap, rubs soap on hands, rinses soap out, turns the water tap off, wipes hands dry, leaves the toilet.

\section{Materials}

A $15.5 \mathrm{~cm} \times 21 \mathrm{~cm}$ folder was made which contained a whole page picture of each separate task put in plastic pockets in the order of performance. The pictures were made with pen and paper. The description of the task was written under each picture, for example "taking the soap".

\section{Procedure}

An $A B A B$ reversal design was employed to evaluate the effects of teaching. During the baseline phase (A) the participant was told "Wash your hands!" No additional help or advice was given. After the hand-washing, he was praised verbally.

Before the training phase began, the trainer taught the participant to use the picture folder. He was told that the folder would help him to remember all of the phases of hand-washing. The participant looked through the picture folder with the trainer who read all the texts aloud. After this, he was asked to read the texts aloud. The whole process took 20 minutes, and it was done twice before the training sessions began. The picture folder was given to the participant, and he was told to look it through before every training session.

In the training phase (B) the participant was first told "Wash your hands". Every correct step of the task analysis was rewarded verbally. If the participant did not respond correctly, a system of least prompts was used to elicit the response (Wolery, Bailey \& Sugai, 1988). The trainer first used verbal prompts, then gestures, and finally the correct response was produced through physical guidance. Follow-up was made two weeks after the training ended.

\section{Data collection and scoring}

Data were gathered during two hand-washing sessions each week day, once in the morning and once in the afternoon. Each of the eight steps was scored from 0 to 3 . The step was scored 3 if it was done independently. It was scored 2, if verbal prompting was used, and it was scored 1 if gestural prompts were needed. The step was scored 0 if the behaviour went wrong or was prompted physically. The sum total across different steps was counted for each session.

\section{Reliability}

Interrater reliability was measured by using a second, independent observer during three sessions. It was calculated by dividing the agreements by the agreements plus disagreements, and by multiplying the quotient by 100 . The mean interrater reliability was $95 \%$.

Procedural fidelity was assessed by observing the teacher during four sessions. Each behavior of the teacher was scored as either right or wrong. Agreement on procedural fidelity was 
calculated by dividing the number of correct trainer behaviors by the number of planned trainer behavior that should have been emitted in the session, multiplied by 100. Mean procedural fidelity was $83 \%$. The ethical standards set by the National Advisory Board on Research Ethics in Finland (2009) were followed in conducting the research.

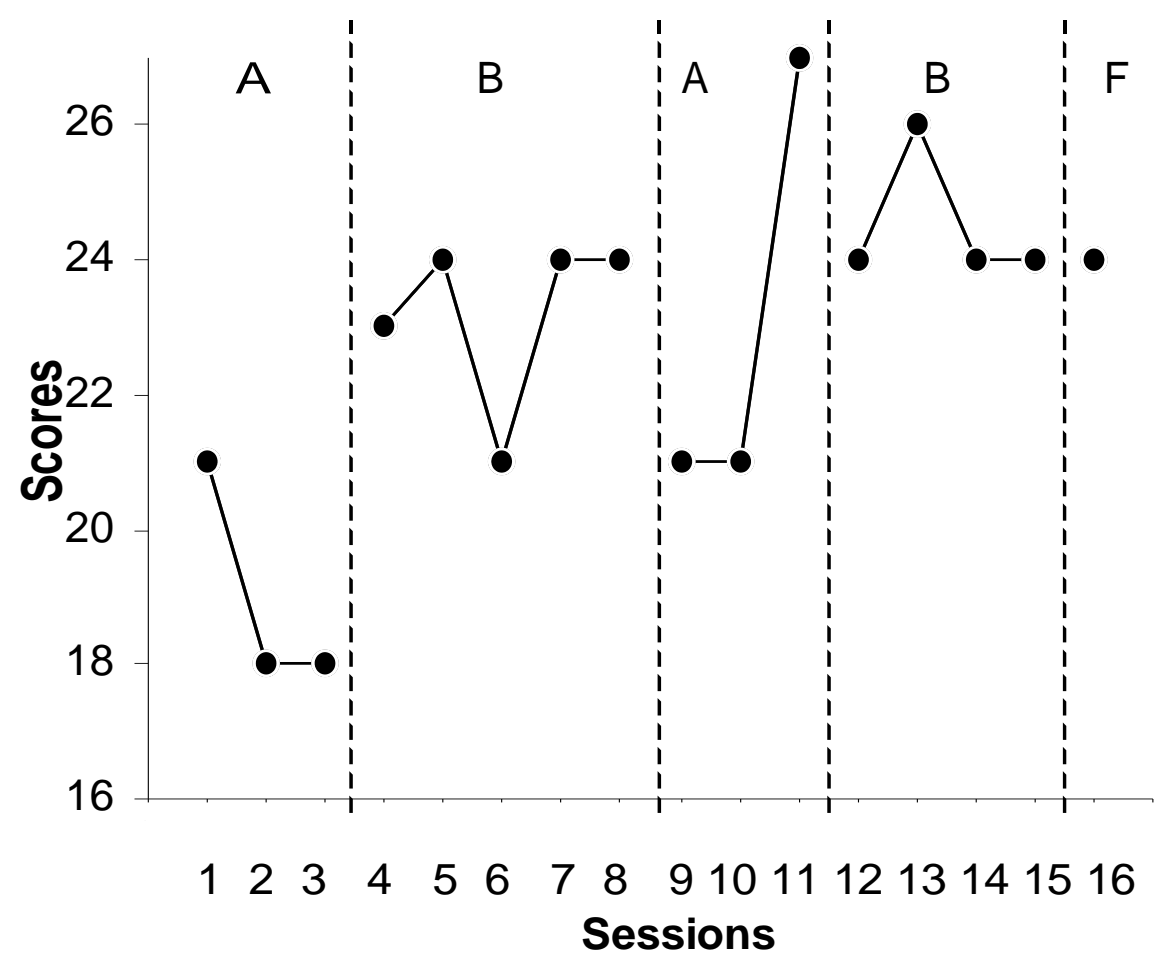

Figure 1. The performance of the participant in hand-washing during the baseline (A), training (B), and follow-up (F).

\section{Results and Discussion}

When the training began, the hand-washing skills of the participant improved immediately when compared with the baseline level (Figure 1). A return to baseline dropped performance, and a subsequent reestablishment of the training phase restored it. A follow-up after two weeks indicated that the obtained results were maintained. The results confirm the effectiveness of pictorial cues in skills acquisition of people with intellectual disabilities. Good results were obtained with the help of a very short period of training, even if the participant still remained dependent on verbal cues on a few tasks.

\section{References}

Alberto, P.A., Cihak, D.F:, \& Gama, R.I. (2005). Use of static picture prompts versus video modeling during simulation instruction. Research in Developmental Disabilities, 26, 327-339

Bambara, L. M. \& Ager, C. (1992). Using self-scheduling to promote self-directed leisure activity in home and community settings. The Journal of the Association for Persons with Severe Handicaps, 17, 67-76.

Browder, D. M. \& Snell, M. E. (1993). Daily living and community skills. In: M. E. Snell (ed.). Instruction of students with severe disabilities. pp. 480 - 525. New York: Merrill.

Caffó, A.O., Albano, V., Damato, C., \& Stella, A. (2013). Autogestione di instruzioni per 
promuovere attivitá funzionali in un bambino con syndrome di Down nel contest classe. Psicologia Clinica dello Sviluppo, 17, 347-358.

Connis, R. T. (1979). The effects of sequential pictorial cues, self-recording, and praise on the job task sequencing of retarded adults. Journal of Applied Behavior Analysis, 12, 355 - 361.

Feldman, M.A. (2004). Self-directed learning of child-care skills by parents with intellectual disabilities. Infants and Young Children, 17, 17-31.

Feldman, M. A., Ducharme, J. M., \& Case, L. (1999). Using self-instructional pictorial manuals to teach child-care skills to mothers with intellectual disabilities. Behavior Modification, 23, 480497.

Kelley, K. R. (2012). Effects of picture prompts delivered by a video iPodrtm on pedestrian navigation. Dissertation Abstracts International Section A: Humanities and Social Sciences, 73.

Lancioni, G.E,,$\underline{\text { Perilli, V }}$, Singh, N.N., O'Reilly, M.F., Sigafoos, J., Cassano,G., Pinto, K., Minervini, M.G., \& Oliva D. (2012). Technology-aided pictorial cues to support the performance of daily activities by persons with moderate Alzheimer's disease. Research in Developmental Disabilities, 33, 265-73.

Martin, J. E., Rusch, F. R., James, V. L., Decker, P. J., \& Trytol, K. A. (1982). The use of pictorial cues to establish self-control in the preparation of complex meals by mentally retarded adults. Applied Research in Mental Retardation, 3, 105-119.

National Advisory Board on Research Ethics in Finland. 2009. The Ethical Principles of Research in the Humanities and Social and Behavioural Sciences and Proposals for Ethical Review. Retrieved from: http://www.tenk.fi/sites/tenk.fi/files/eettisetperiaatteet.pdf

Phillips, C. L., \& Vollmer, T.R. (2012). Generalized instruction following with pictorial prompts. Journal of Applied Behavior Analysis, 45, 37-54.

Thinesen, P. J. \& Bryan, A. J. (1981). The use of sequential pictorial cues in the initiation and maintenance of grooming behaviors with mentally retarded adults. Mental Retardation, 19, $247-250$.

West, E.A. (2008). Effects of verbal cues versus pictorial cues on the transfer of stimulus control for children with autism. Focus on Autism and Other Developmental Disabilities, 23, 229-241.

Wolery, M., Bailey, Jr., D. B. \& Sugai, G. M. (1988). Effective teaching. Principles and procedures of applied behavior analysis with exceptional students. Boston: Allyn and Bacon. 